\title{
LA CONCEPCIÓN ESTRUCTURAL DE LA CIENCIA. UNA LECTURA HISTÓRICA DESDE SUS APORTES A LA PRAGMÁTICA
}

\author{
Adriana Gonzalo* \\ Universidad Nacional del Litoral / CONICET \\ (Argentina)
}

\section{Resumen}

El presente trabajo tiene como objetivo central realizar aportes a la historia de la filosofía de la ciencia en general, y de la Concepción Estructural (CE) en particular, resaltando las contribuciones que desde esta última se han realizado en el campo de la pragmática; como también evaluar dichos logros, y señalar los límites y potencialidades de las propuestas analizadas en marco de la CE. En una primer etapa se comentarán los primeros desarrollos de las ideas pragmáticas en la CE, en integración con la propuesta kuhniana (Sneed 1971; Stegmüller 1973, 1976, 1978, 1979); para proseguir con los desarrollos posteriores en esta línea de trabajo (Moulines 1982, 1991a, 1991b, 2002); Balzer, Moulines, y Sneed 1987). Finalmente, se evaluará el "intento conciliatorio" entre enfoques socio-históricos y enfoques formales en relación a los aportes a una pragmática de la ciencia dentro de la CE y se cuestionará la visión de la "historia heredada" de la filosofía de la ciencia.

Palabras claves: Concepción Estructural, pragmática de la ciencia, historia de la filosofía de la ciencia

\footnotetext{
Abstract

The central objective of the present paper is to make contributions to the history of the philosophy of science in general and to the Structuralist View (SV) in particular, highlighting the contributions made in the field of pragmatics from the latter. It is also a purpose to

Recibido: 23/02/2011. Aceptado: 16/05/2012.

* Este trabajo ha sido realizado con la ayuda de los proyectos de investigación PICTR $2006 \mathrm{~N}^{\circ}$ 2007 y PICT2007 No 1558 de la Agencia Nacional de Promoción Científica y Tecnológica (Argentina) y FFI2008-01580 y FFI2009-08828 del Ministerio de Ciencia e Innovación (España). Agradezco a José A. Díez y a José L. Falguera las sugerencias y comentarios realizados a una versión anterior, más extensa, del presente trabajo.

* e-mail: adriana.n.gonzalo@gmail.com
} 
evaluate the achievements and to point out the limits and potentialities of the proposals argued in the SV framework. In a first stage the early developments of pragmatic ideas in the SV and its integration with the Kuhn's perspective are discussed (Sneed 1971); (Stegmüller 1973, 1976, 1978, 1979). The work continues with the subsequent developments in this line of work (1982 Moulines, 1991a, 1991b, 2002); (Balzer, Moulines, and Sneed 1987). Finally, the "conciliatory attempt" of socio-historical approaches and formal approaches in relation to the proposals in the pragmatic of science within the SV are evaluated, and the view of the "inherited history" of the philosophy of science is discussed.

Keywords: Structuralist View, pragmatic conception of science, history of the philosophy of science

\section{Introducción}

1.1. La Concepción Estructural de las teorías científicas (CE) constituye una de las concepciones semánticas o modelo-teóricas de la ciencia ${ }^{1}$, y desde sus comienzos hasta la actualidad ha ido desarrollando un marco conceptual que corresponde al ámbito metateórico general y que, a su vez, posibilita abordar estudios epistemológicos y reconstrucciones de casos puntuales de la historia de la ciencia.

Así, en el marco de la CE se han desarrollado trabajos concernientes a la organización interna de las teorías y reconstrucciones racionales, basados en herramientas formales, en especial en procedimientos de reconstrucción conjuntista; como así también otros que se orientan a los componentes histórico-pragmáticos y a las reconstrucciones diacrónicas de las teorías científicas. $^{2}$

Si bien en el seno de las publicaciones propias de la CE los trabajos en ambas direcciones se encuentran claramente representados; en general, no es frecuente que los aportes que desde la CE se han realizado en relación a los

${ }^{1}$ Las concepciones semánticas o modelo-teóricas de la ciencia conforman una "familia", que comparte algunos elementos generales. A esta familia, además de la CE, pertenecen P. Suppes, B. van Fraassen y F. Suppe (seguidores de los trabajos pioneros de J. McKinsey, E. Beth y J. von Neumann), además de R. Giere, en los Estados Unidos; M. Dalla Chiara y G. Toraldo di Francia, en Italia; M. Przelecki y R. Wójcicki, en Polonia.

${ }^{2} \mathrm{La}$ CE abarca una etapa fundacional con la obra de J. Sneed (1971) y el desarrollo siguiente en W. Stegmüller (1973); y etapas posteriores, donde se suman las colaboraciones de sus discípulos principales: C.U. Moulines y W. Balzer. Con estos últimos, y nuevos aportes de sus fundadores, la CE por un lado, amplía el aparato metateórico inicial, y por otro extiende su ámbito de aplicación hacia diversas disciplinas científicas. Gran parte de los resultados de esta etapa se recogen en Balzer, Moulines \& Sneed (1987). Asimismo, la comunidad filosófica que integra la CE continúa hasta la actualidad, con un continuo aporte de aspectos conceptuales que contribuyen a la elucidación metateórica, como de numerosos trabajos de reconstrucción epistemológica. (Una bibliografía muy completa de esos trabajos se encuentra en Diederich, Ibarra \& Mormann (1989), Diederich, Ibarra \& Mormann (1994)). 
aspectos histórico-pragmáticos de la ciencia y la reflexión metateórica sobre la actividad científica, sean igualmente conocidos y estudiados.

El presente trabajo tiene como objetivo central realizar aportes a la historia de la filosofía de la ciencia en general, y de la CE en particular, resaltando las contribuciones que desde esta última se han realizado en el campo de la pragmática; como también evaluar dichos logros, y señalar los límites y potencialidades de las propuestas analizadas.

Un objetivo más general es contribuir, mediante el análisis indicado, a una revisión de lo que podríamos llamar la "historia heredada" de la filosofía de la ciencia $(\mathrm{HH}){ }^{3}$ Plantearé que en el marco de la misma, se ha sostenido la idea de ruptura o cambio revolucionario entre un período prekuhniano y uno postkuhniano, que contribuyó a acentuar una serie aspectos antagónicos entre los períodos y posiciones situadas en los mismos. Algunos de éstos serían: (a) la consideración de las "teorías" científicas (como entidades enunciativas, estructuradas lógicamente) como objeto del análisis epistemológico, versus la consideración de unidades más amplias ("estilos de pensamiento" en el caso de Fleck (1935); "paradigmas" o "matrices disciplinares”, en el caso muy conocido de Kuhn (1962, 1969)); (b) enfoques estáticos, y elucidaciones sobre la estructura y componentes teóricos, versus enfoques dinámicos de la ciencia, que contemplen factores sociológicos y culturales constitutivos del estudio de casos de la historia de la ciencia; (c) la atención a los aspectos lógico-semánticos y metodológicos de las teorías científicas, versus el interés por los componentes socio-pragmáticos de la actividad científica; (d) las reconstrucciones formales de las teorías científicas, versus las reconstrucciones histórico-sociológicas de la ciencia.

Defenderé aquí la idea de que la CE representa un enfoque filosófico que posibilita revisar la lectura del giro-sociohistórico, presentado como una ruptura inconciliable con los enfoques previos, generalmente englobados bajo la denominación de enfoques formales o lógico-semánticos de la ciencia.

1.2. La estrategia a seguir en el trabajo será la siguiente:

i) se mostrará cómo la CE, desde sus propios inicios, no se presenta como una alternativa semántico-formal en oposición al protagonista indiscutido del giro-sociohistórico, T. Kuhn; sino que, por el contrario, propone

${ }^{3}$ Esta "historia heredada" tiene como referencia principal la obra de Suppe, F. (1974), que ha sido seguida por muchos autores, entre ellos Brown, H. (1977 y; Hacking, I., ed. (1981). Una imagen de la historia de la ciencia en la misma dirección se ha consolidado en muchos de los escritos de sociología de la ciencia: Bloor, D. (1976); Barnes, B. (1976); Hesse, M. (1980). 
un diálogo integrativo con éste. Así, una primera parte del trabajo consistirá en presentar de modo genérico y en analizar las principales ideas de la CE en esta integración con la posición kuhniana.

ii) Seguidamente, siguiendo siempre el objetivo de presentación histórica de la CE, se comentarán los primeros desarrollos de las ideas pragmáticas en la CE, en integración con la propuesta kuhniana (Sneed 1971; Stegmüller 1973, 1976, 1978, 1979); para proseguir en el punto siguiente, con los desarrollos posteriores en esta línea de trabajo (Moulines 1982, 1991a, 1991b, 2002; Balzer, W., Moulines, C. U. y Sneed, J. 1987).

iii) A continuación, se evaluará este "intento conciliatorio" entre enfoques socio-históricos y enfoques formales en relación a los aportes a una pragmática de la ciencia dentro de la $\mathrm{CE}$, a la luz de los desarrollos actuales, evaluando sus aportes y señalando sus limitaciones.

iv) Finalmente, como consecuencia de lo anterior, se intentará abrir una serie de discusiones contra la $\mathrm{HH}$, que como comentamos defiende una línea de ruptura o cambio radical en los enfoques y ejes de análisis producidos desde el giro sociohistórico; y sostiene antinomias irreconciliables entre los enfoques históricos y los enfoques formales de la ciencia.

\section{Introducción: Kuhn y la Concepción Estructural de la ciencia}

La interpretación metateórica de Kuhn desde la CE fue desarrollada en relación a variados aspectos de la reflexión sobre la ciencia: la noción de ciencia normal, la idea de cambio científico y la noción de conmensurabilidad local, aspectos ontosemánticos de la ciencia —significado de los términos científicos, holismo semántico, etc.- diversas nociones en relación a componentes socio-pragmáticos, en especial: la de comunidad científica, la idea de "disponer de una teoría", la de acciones intencionales en la práctica científica.

Desde los textos originales de la CE antes citados, una vasta cantidad de estos tópicos han sido abordados y analizados por diversos autores ${ }^{4}$, quienes han incursionado en muchos de las temáticas arriba listadas. Dados los objetivos mencionados en (1), nos centraremos acá solamente en los aspectos comentados allí, que servirán como ejes a discutir posteriormente.

${ }^{4}$ Entre la múltiple bibliografía reciente dentro de la CE, podemos citar: Falguera, J. L. (1999, 2004, 2009) Jaramillo, J. M. (1996); Lorenzano, C. y Lorenzano, P. (1996); Lorenzano, P. (2008). 
En el prólogo de Stegmüller (1973), el autor comentaba que la lectura de la primera parte de ese libro podía emprenderse bajo dos puntos de vista completamente distintos: "Por una parte, como intento de justificar, y también de aplicar en sus aspectos más importantes, un procedimiento completamente nuevo para el análisis de la estructura de las teorías científicas. Por otra, esta parte proporciona los instrumentos conceptuales necesarios para el análisis de la dinámica de las teorías y para la reconstrucción lógica de los conceptos "ciencia normal" y "revoluciones científicas" de T. S. Kuhn". (Stegmüller: 1973, p. 9)

A lo largo de la obra, en lo que respecta al análisis de la posición kuhniana, la actitud de Stegmüller (1973) es claramente conciliadora. Sostiene: “...si bien los desarrollos presentados a continuación pueden aparecer frecuentemente en forma y en contenido, muy polémicos y desafiantes, no debería olvidarse que van del todo dirigidos a una reconciliación entre los lógicos de la ciencia por un lado, y los rebeldes contra la filosofía de la ciencia, por otro. Que la reconciliación tenga lugar o no, depende, entre otras cosas, de si ambas partes están dispuestas a hacer concesiones." (Stegmüller 1973, p.12).

Los aspectos que en Stegmüller (1973) se ponen en común entre ambos enfoques, son básicamente los siguientes:

a) El autor, al presentar como alternativa a una "concepción enunciativa" de la ciencia una concepción estructuralista, se opondrá a aquella concepción del conocimiento científico, reconociendo que las insuficiencias marcadas por Kuhn a la misma podían claramente ponerse en conciliación con su propia visión de la ciencia. ${ }^{5}$

b) La introducción de la dinámica de las teorías como temática central de la agenda filosófica del estructuralismo aparecía, entonces, como una respuesta al reto kuhniano. A diferencia de otros autores, como Lakatos $(1970,1971)$, quienes consideraban que la posición kuhniana de cambio teórico conducía a sostener posiciones irracionalistas (recordemos que el ataque central se da a la noción de conversión por un lado, y a la de inexistencia de pautas metodológicas y gnoseológicas que permitan dar cuenta de la elección teórica por otro); el estructuralismo aceptará la necesidad

${ }^{5}$ Afirma Stegmüller: "Una de las ideas fundamentales de la lógica y de la metamatemática modernas es la idea de que las teorías son determinados sistemas o clases de enunciados. Las investigaciones se concentran en el estudio de las relaciones de deducción lógica que existen entre los elementos de estas clases. Llamaré a este tipo de consideración el modo de consideración micrológico y a la concepción de las teorías como clases de enunciados la llamaré statement view de las teorías o concepción enunciativa de las teorías". (Stegmüller 1973, p. 18 —se reproduce el uso de itálicas originales del texto-) 
de explicar el cambio teórico no sólo sobre consideraciones justificatorias lógico-metodológicas, sino —en consonancia parcial con Kuhn- sobre consideraciones pragmáticas.

c) En relación al mote de "científico irracional" aplicado al actor del cambio teórico del paradigma kuhniano, Stegmüller (1973) pensaba que la negación de Kuhn de concebir el cambio teórico en el sentido progresivo tenía un aspecto adecuado; y era conveniente considerar que "el modo de pensar en relaciones de deducibilidad entre enunciados es un método totalmente inadecuado para comparar entre sí la teoría suplantadora y la suplantada”. (Stegmüller 1973, p.11). Admitía además que la vía kuhniana de mostración de ejemplos era claramente transitable como alternativa a la visión enunciativa del cambio teórico, particularmente sobre la propuesta de reducción teórica planteada desde aquella. El análisis filosófico de la ciencia debía integrarse a los análisis históricos. Debía aprenderse de estos últimos la necesidad de dejar de hacer un análisis genérico y descontextualizado de la ciencia, y considerar las teorías como objetos concretos, ubicados histórico-culturalmente.

En la misma línea, Moulines, C. U. (1982, 1991a, 1991b) reconoce a T. Kuhn como quien encarna una crítica a la filosofía tradicional, pero al mismo tiempo estima que la filosofía de aquel no debe interpretarse tanto como una filosofía de la ciencia alternativa, sino como el reto filosófico de un historiador a los filósofos de la ciencia profesionales: el reto de exigir a los filósofos un aparato conceptual preciso que dé cuenta de los aspectos de las ciencias empíricas, que Kuhn había revelado en sus análisis históricos.

Moulines (1982) al desarrollar "Un programa de reconstrucción estructural de las teorías físicas", presenta una serie de tesis que estima como los aspectos realmente cruciales revelados por Kuhn. Dentro de éstas, en consonancia con (a) arriba, el autor remarca que Kuhn había señalado que "Una teoría empírica es algo más que un conjunto de hipótesis o axiomas". Asimismo, en relación con (b) arriba, señala que "Las aplicaciones empíricas de una teoría forman parte especial del concepto mismo de teoría. La estructura teórica y sus aplicaciones están conceptualmente ligadas de manera estrecha". "Una teoría empírica es una entidad que evoluciona en el tiempo; esta evolución es lo que Kuhn llama «ciencia normal»". Y agrega posteriormente: "Las revoluciones científicas son eventos en los que una teoría sustituye completamente a otra, con profundos cambios conceptuales y sin que medie una relación lógica entre ambas teorías". Moulines resalta cómo estas tesis habían provocado - por entonces- una década de fuertes polémicas en el mundo filosófico, y agrega: "Ellas parecerían a mucha gente incompatibles no sólo con las concepciones metodológicas usuales, sino 
también con cualquier programa de reconstrucción racional de la empresa científica". Sin embargo — según el autor-, lo que Kuhn había rechazado era una visión simplista de la ciencia, concebida como conjunto de axiomas cuyas consecuencias lógicas podían confirmarse o rechazarse, mostrando la necesidad de una concepción más compleja y profunda de las teorías científicas. (Moulines (1982, pp.76-77)).

Vemos así como, en consonancia con Stegmüller (1973), Moulines (1982) mantiene un carácter similar en la presentación de la CE, en la que se muestra a ésta explícitamente conectada con la visión kuhniana de ciencia, en particular de ciencia normal. A su vez, estas dos obras presentan una serie de tesis - a modo programático- que señalan la posibilidad de conciliar varios de los conceptos kuhnianos con los aspectos formales de la CE; al mismo tiempo que se enfatizan los elementos pragmáticos como comunes de ambas propuestas metateóricas.

\section{La CE: concepción de teoría y componentes pragmáticos. Una integra- ción con la propuesta kuhniana. Primeros desarrollos}

\subsection{Los aportes de Sneed (1971):}

La primera aproximación que Sneed establece explícitamente con la concepción kuhniana de ciencia se realiza a través del acercamiento entre de la noción de "teoría" propuesta en Sneed (1971) y a la idea de "ciencia normal”.

Como es bien conocido, Sneed (1971) presentó su propuesta sobre la estructura formal de las teorías sosteniendo que pueden distinguirse rasgos esenciales de éstas, que se asocian a una estructura formal, matemática $(K)$, y a un conjunto de aplicaciones intencionales $(I)$, de modo que una teoría (T) puede representarse mediante la tupla $<K, I>$. El componente $K$ incluía en (Sneed 1971) ${ }^{6}$ el conjunto de los modelos $(M)$, el de los modelos potenciales $\left(M_{p}\right)$, el conjunto de Constraints $(C)$, y el conjunto de los modelos potenciales parciales $\left(M_{p p}\right)$. Así, $K=<M_{p}, M, M_{p p}$, C>.

Recordemos que: $M p$ simboliza la clase total de entidades que satisfacen las condiciones que caracterizan matemáticamente al aparato conceptual de la teoría y son aquellas estructuras de las cuales tiene sentido preguntarse si son modelos, pero de las cuales todavía no se sabe si efectivamente lo son;

${ }^{6}$ Como veremos luego, los componentes formales de K serán precisados y desarrollados en mayor extensión posteriormente, particularmente en Sneed (1976), Balzer, Moulines y Sneed (1987). 
mientras que $M$ simboliza a las entidades que satisfacen la totalidad de las condiciones introducidas, es decir, que además satisfacen las leyes fundamentales o "axiomas propios" de la teoría en cuestión.

Para nuestros propósitos nos interesa añadir que los $M_{p p}$ de una teoría $T$ se conciben como lo que podría llamarse la "base empírica" de $T$. Este conjunto de entidades quedaba determinado por un predicado conjuntista definido por los mismos axiomas estructurales que determinan $M_{p}$, a excepción de aquellos que se refieren a los términos teóricos relativos a $T$ (o $T$-teóricos). Debemos resaltar este aspecto de que los $M_{p p}(T)$ son sistemas a los que se ha "podado" de dichos términos $T$-teóricos y son caracterizables mediante los otros términos (los T-no-teóricos). ${ }^{7}$

Informalmente, las ideas sneedianas podrían básicamente expresarse así: tenemos por un lado el núcleo matemático de una teoría $(K)$; y, además, determinados sistemas físicos, que conforman un subconjunto de $M p p$, el conjunto de las aplicaciones propuestas $(I)$ características de la teoría. Para que esto sea posible, es necesario que se hayan conceptualizado estos sistemas a los que se desea aplicar el formalismo del núcleo en términos compatibles con la conceptualización presentada en éste. Considerándolos, por ende, tales sistemas como estructuras del tipo de los $M_{p p}$ de la teoría, es decir, estructuras que son compatibles con la teoría, pero que no la presuponen. Diferenciada de la teoría $T=<K, I>$, pero asociada a ella, disponemos de la afirmación $I \in C n(K)$ que es conocida como "la aserción empírica central", donde $\mathrm{Cn}$ es un operador modelo-teórico que aplica estructuras completas $T$-teóricas de la teoría a estructuras parciales ( $T$-no-teóricas) para establecer el contenido empírico de $T$.

Finalmente, Sneed incorpora en la caracterización de $K$ la idea de constraints $(C)$, a partir de considerar que, además de las condiciones que afectan a cada modelo, debían contemplarse otras condiciones que se establecen entre diferentes modelos de una teoría, de manera que algunos éstos estarían interconectados formalmente conformando una estructura global. Así, se propuso, para incorporar esta idea al formalismo caracterizador de una teoría, las constraints entre las funciones correspondientes a los diversos modelos de una teoría.

Al mismo tiempo que desarrollaba las bases formales para una axiomatización sincrónica de las teorías físicas, Sneed bosquejaba un proyecto de

${ }^{7} \mathrm{La}$ distinción entre términos T-teóricos y T-no-teóricos responde a lo que se ha denominado "criterio de teoricidad de Sneed": un término $t_{i}$ de $T$ es T-teórico si en todas las determinaciones de $t_{i}$ las partes de la teoría usada (presupuesta) por los científicos con el propósito de su determinación son parte o coinciden con T. (Sneed, 1971) 
acercamiento entre una concepción modelo-teórica y las ideas de cambio científico y ciencia normal kuhniana, contribuyendo a lo que se ha llamado una "racionalización" de las ideas kuhnianas. ${ }^{8}$

Sneed, valiéndose de la noción de "núcleo expandido" de una teoría, había postulado que en el transcurso del desarrollo de una teoría, el núcleo matemático de la teoría permanece estable, mientras el conjunto de aplicaciones propuestas varía. Esta noción se basa en la idea de que, si al núcleo matemático inicial de una teoría se suman algunas leyes especiales y constraints, el conjunto de aplicaciones de la teoría varía, mientras que el núcleo original (las leyes fundamentales de la teoría) permanece invariante. Sneed sugirió que esta idea de desarrollo de una teoría podía ponerse en concordancia con la idea de "ciencia normal" de Kuhn.

Al mismo tiempo, Sneed había introducido la noción de disponer de una teoría, en el sentido de que en un período histórico de desarrollo de una teoría, se dispone de un conjunto determinado de aplicaciones paradigmáticas del núcleo, que forman parte del conjunto total de aplicaciones de éste, sobre la base del cual se producen los futuros cambios en dicho conjunto. Así, señalaba el carácter intencional de las aplicaciones y mostraba correlativamente el aspecto pragmático involucrado, al aducir a las actitudes de los científicos que disponen de una teoría $T$ en un tiempo $t$ con el que operan en sus prácticas científicas.

Sobre estas ideas, Stegmüller (1973) presentó un extenso desarrollo y una generalización de las ideas sneedianas.

\section{2.- La propuesta de Stegmüller (1973, 1976, 1978):}

3.2.1.- En Stegmüller (1973) se profundiza la distinción sneediana entre teoría y permanencia o estabilidad de una teoría; y enunciado o aserción empírica de una teoría. Se recalca que, mientras el núcleo de una teoría permanece estable, las aplicaciones del núcleo pueden variar históricamente con los enunciados empíricos centrales de la teoría. Stegmüller afirmaba que: "Si la teoría física consiste en el par $<K, I>$ y $E_{1}, E_{2}, \ldots E_{i}, \ldots$ son las diversas ampliaciones de $K$ empleadas en momentos diversos $t_{1}, t_{2}, \ldots$ $t_{i}$, ... para establecer aserciones [empíricas ...], esto se puede describir más exactamente usando la operación de aplicación $A_{e}$, sin recurrir en absoluto a entidades lingüísticas: la teoría $<K, I>$ sigue siendo la misma; las proposiciones de teoría $I \in A_{e}\left(E_{i}\right)$ construidas con su ayuda y que se consideran válidas varían con el tiempo $t_{i}$." (Stegmüller 1973, p.241).

${ }^{8}$ El mismo Kuhn ha reconocido que la interpretación estructuralista de sus ideas centrales le parecían absolutamente conciliables con su propia concepción (Kuhn, 1976). 
Con la ayuda de estas nociones, Stegmüller irá precisando el concepto de "disponer de una teoría", tal y como inicialmente se expresa en la siguiente definición (I):

Def. 1:

La persona $p$ dispone en el sentido sneediano de una teoría física $T=\langle K$, $I>$ en el instante $t$ syss:

(1) $<K, I>$ es una teoría física en el sentido de Sneed;

(2) existe una ampliación $E_{t}$ de $K$ tal que $p$ cree en el instante $t$ que $I \in A_{e}\left(E_{t}\right)$. Esta ampliación es la ampliación más restrictiva de $K$, en el sentido de que se cumple: $\Lambda E\left[\left(E\right.\right.$ es una ampliación de $K$, tal que $p$ cree cuando $t$ que $I \in A_{e}(E)$ y $p$ dispone de datos observacionales que apoyan esta proposición) $\left.\rightarrow E_{t} \subseteq E\right]$;

3) $p$ dispone de datos observacionales que apoyan la proposición $I \in A_{e}\left(E_{t}\right)$;

4) p cree en el instante $t$ que existe una ampliación $E$ de $K$ que cumple:

a) $I \in A_{e}(E)$

b) $A_{e}(E) \subset A_{e}\left(E_{t}\right)$. (Stegmüller 1973, p. 243)

Una vez admitida la noción de teoría $T$ de Sneed (1), tenemos que: (2) nos dice que una persona $p$ cree que la aserción empírica de $T, I \in A_{e}\left(E_{t}\right)$, es la de mayor contenido empírico (en el conjunto de todas las aserciones de $T$ que corresponden a lo que cree); (3) establece que la convicción de creencia de $p$ en un tiempo $t$ supone que $p$ dispone de algún tipo especial de elementos de juicio para creer en una proposición de la forma $I \in A_{e}\left(E_{t}\right)$, lo que equivale a sostener que tal creencia viene apoyada por datos observacionales de que dispone la persona; y finalmente, la condición (4) expresa que $p$ cree que el conjunto de las aplicaciones intencionales de una teoría en un tiempo $t_{i}$ será mayor (más amplio) en un $t_{j}(\mathrm{j}>\mathrm{i})$.

A partir de esta definición de la noción de "disponer de una teoría en el sentido de Sneed", Stegmüller avanza sobre condiciones complementarias para poder integrar esta noción al marco metateórico kuhniano. Para esto, en Stegmüller (1973) se enfatiza la existencia de componentes estables o invariantes en el período de ciencia normal kuhniano, noción que se redefine en lectura estructuralista, resaltando la noción de núcleo estructural, en primer lugar, y en segundo, la noción de $I_{0}$ de ejemplos paradigmáticos. Se estima que este último fue considerado por Kuhn como primordial en la determinación de la identidad de una teoría a lo largo del tiempo. A este último componente se sumaban las nociones ya antes introducidas previamente (Def. I). Con estos componentes la reconstrucción estructuralista de "disponer de una teoría en el sentido kuhniano" se presentaba en la siguiente definición (II):

Def. II:

La persona $p$ dispone en el instante $t$ en el sentido de Kuhn de una teoría física $T=<K, I>$ syss se cumple: 
1) $<K, I, I_{0}>$ es una teoría física en el sentido de Kuhn;

2) existe una ampliación $E_{t}$ de $K$ tal que $p$ cree en el instante $t$ que $I \in A_{e}\left(E_{t}\right)$. Esta ampliación es la ampliación más restrictiva de $K$, en el sentido en que se cumple: $\Lambda E\left[\left(E\right.\right.$ es una ampliación de $K$, tal que $p$ cree cuando $t$ que $I \in A_{e}(E)$ y $p$ dispone de datos observacionales que apoyan esta proposición) $\left.\rightarrow E_{t} \subseteq E\right]$;

3) $p$ escoge $I_{0}$ como conjunto de ejemplos paradigmáticos para I;

4) $p$ cree cuando $t$ que $\Lambda t^{*}$ (si $I^{p}{ }_{t}$, es el conjunto de las aplicaciones de la teoría física $<K, I>$, en el sentido de Sneed, entonces $I \subseteq I_{t^{\prime}}^{p}$ );

(5) $p$ dispone cuando $t$ de datos observacionales que apoyan la proposición $I \in A_{e}$ $\left(E_{t}\right) ;$

(6) p cree cuando t que existe una ampliación $\mathrm{E}$ de $\mathrm{K}$ tal que:

a) $I \in A_{e}(E)$

b) $A_{e}(E) \subset A_{e}\left(E_{t}\right) \cdot($ Stegmüller 1973, p. 277)

Como es evidente, las condiciones (1), (2), (5) y (6) son coincidentes con las (1) a (4) de nuestra Def. (I). La condición (3) expresa que la persona $p$ admite $I_{0}$ como conjunto de aplicaciones paradigmáticas, contenidas en $I$; mientras que (4) impone que $I_{0}$ será siempre un subconjunto del conjunto total de aplicaciones de $T$ en un tiempo futuro.

Con este marco desarrollado, el autor estima que la integración con Kuhn se manifiesta en que se recupera el origen histórico de $T$, a través del componente de ejemplos paradigmáticos originarios. Éstos se conservan a lo largo del desarrollo de la teoría, a pesar del cambio sucesivo de aplicaciones intencionales de $T$. Por otro lado, se sigue preservando la idea de núcleo estructural fijo, al modo de los aspectos centrales del paradigma en un momento de ciencia normal kuhniana. Así, con esta definición los cambios teóricos ocurridos en un período de ciencia normal, se expresan en términos de aserciones empíricas de una teoría $T$ y cambios en las aplicaciones de $T$, manteniéndose estable tanto $K$, como el conjunto de ejemplares de $T$.

3.2.2. A partir de Sneed (1971) hasta comienzos de los años 80, van a producirse ciertos cambios en la caracterización inicial de "teoría" en la $\mathrm{CE}$, que producirán variaciones en relación a la vinculación con la perspectiva kuhniana.

Como sabemos, lo que originalmente fue concebido bajo la denominación simple de teoría, comenzó a denominarse "elemento teórico". La caracterización de esta entidad siguió básicamente identificándose con la de teoría de Sneed (1971), comentada antes. ${ }^{9}$ Sumada a esta noción, se

${ }^{9}$ La definición de "elemento teórico" ha sufrido diversas precisiones a lo largo del desarrollo de la CE, hasta su versión actual: un elemento teórico consiste del núcleo $K$ y el campo de aplicaciones propuestas o intencionales $I: T=\langle K, I\rangle$, donde $K=\langle M p, M, M p p$, $G C, G L\rangle . M p$ es conjunto de modelos potenciales de $T, M$ simboliza los modelos actuales (o 
propuso hablar de "red-teórica", para referirse a una teoría en sentido más amplio, equivalente a un conjunto de elementos teóricos que deben satisfacer una serie de condiciones. Se había pensado originalmente que el conjunto de los modelos representaba las leyes fundamentales de una teoría (en el sentido de elemento teórico), que se cumplen en todas las aplicaciones de ésta. Las leyes especiales y las constraints especiales en relación al "núcleo básico" de la teoría, constituían lo que se denominó "núcleo expandido". La dinámica de las teorías se describía en términos de núcleos expandidos (que cambiaban el ámbito de las aplicaciones de la teoría), manteniéndose el núcleo firme de ésta. El cambio central se manifestó al considerar que la presencia de leyes especiales podría dar lugar a la formación de subteorías o teorías surgidas a partir de una teoría principal (elemento-básico), que contendría las leyes fundamentales o núcleo firme. ${ }^{10}$

En el marco de los cambios comentados, en Stegmüller (1976), se presentan algunas variaciones de la propuesta con respecto a Stegmüller (1973). Entre éstas se encuentra la incorporación de la idea de red teórica $\mathrm{N}$ y la de $N^{*}$ de núcleo de red; tomando ahora la conceptualización siguiente la forma:

"La aserción de que una persona $p$ dispone de una teoría $T[\ldots]$ en un tiempo $[\ldots]$ significa que hay una red $N$ basada en especializaciones del núcleo $K$ de $T$, tal que $p$ cree que $I \in A\left(N^{*}\right)$ en $t$, y también que $p$ tiene evidencia de apoyo para esta proposición, y finalmente que $p$ cree que $N$ es la red existente más fuerte tal que $I \in A\left(N^{*}\right)$." (Stegmüller, 1976, p. 223)

El autor agrega que, puede además sumarse otra cláusula que indique que la persona $p$ cree en el progreso teórico, y de este modo se afirmaría:

sencillamente modelos de la teoría); $M p p$ es el conjunto de modelos potenciales parciales, o la base empírica de T. GC (Global Constraints) es el conjunto de constrainsts o condiciones de ligadura, que conectan de determinadas maneras fijas los valores que pueden tomar las funciones correspondientes de los diversos modelos; mientras que GL (Global Links) simboliza el conjunto de links o vínculos interteóricos. (Balzer, Moulines y Sneed, 1987).

${ }^{10}$ Afirma Sneed: "Ahora creo que hay una manera un tanto más perspicua, pero equivalente, de describir esos rasgos de las teorías. Primero defino la noción de "especialización" de un núcleo teórico. Intuitivamente, una especialización de $K=<M_{p}, M_{p p}, M, C>$ asigna a algún subconjunto de $\mathrm{M}_{\mathrm{pp}}$ ciertas leyes especiales que representan restricciones adicionales sobre el conjunto $M$, junto con algunas constraints asociadas a esas leyes - que representan restricciones ulteriores sobre $C$. Si $T$ y $T$ ' son elementos teóricos, entonces $T$ ' es una especialización de $T$ syss: (1) $M^{\prime}{ }_{p p} \subseteq M_{p p} ;(2) \operatorname{Po}(M) \cap A(K) \neq \phi$; (3) $M^{\prime}=\left\{x / x \in M_{p}\right\}$ y r $(x) \in M$; (4) $M^{\prime} \subseteq M$; (5) $C^{\prime} \subseteq C$; y $I^{\prime}=I \cap M_{p p}^{p}$." (Sneed, 1976). Aclaración: El significado de $A(K)$ puede indicarse como sigue: dado un núcleo teórico $K=<M_{p}, M_{p p}, M, C>$ se selecciona una clase de subconjuntos de $M_{p p}$ según el siguiente criterio: un subconjunto de $M_{p p}$ está en $A(K)$ syss puede agregarse a cada miembro de él componentes teóricos, de manera tal que se produzca un subconjunto $M$ (que satisfaga las leyes teóricas) y tal que el total de los componentes teóricos satisfagan las constraints. 
“ $p$ cree en $t$ que hay una especialización $K s$ ' de $K s$ que no está aún en la red $N$, y que puede dar como resultado una red más fuerte $N^{\prime}$ tal que $I \in A\left(N^{* \prime}\right)$ y $A\left(N^{*^{\prime}}\right) \subset A$ $\left(N^{*}\right)$.” (Stegmüller 1976, p. 223)

En esta variante de la conceptualización original Stegmüller afirma que puede interpretarse ahora que "disponer de una teoría en el sentido de Kuhn” es equivalente a pertenecer a la misma tradición científica. Así, personas pertenecientes a un grupo de científicos en un tiempo $t$ usan $K$ y éste permanece inalterable; mientras que las aserciones empíricas cambian sucesivamente según las aplicaciones intencionales de $K$. El cambio teórico así descripto corresponde a lo que en Stegmüller (1976) se denomina "cambio accidental" de teoría.

Una versión similar a la presentada antes, aparecía también en Stegmüller (1978). El autor sostiene la siguiente definición:

Def. III:

"Que una persona p dispone en el tiempo $t$ de una teoría $T$ con la aplicación intencional I puede ahora ser explicada aproximadamente por la satisfacción de las condiciones siguientes:

(1) Existe una persona (o un grupo de personas) $p_{0}$ que ha determinado el núcleo básico $K_{b}$ y el conjunto $I_{0}$ de ejemplos paradigmáticos $I_{0}$;

(2) $p_{0}$ por primera vez ha aplicado con éxito una red $N$ bajo $K_{b}$ a un conjunto $I$, con $I_{0} \subseteq I$

(3) $p$ acepta el conjunto $I_{0}$ de ejemplos paradigmáticos;

(4) $p$ sabe en $t$ que una red teórica $N$ con base $B(N)=\mathrm{T}_{\mathrm{b}}=\left\langle\mathrm{K}_{\mathrm{b}}\right.$, I $>$ y un conjunto $I$, con $I_{0} \subseteq I$ y sabe de $I$ y de un núcleo de la red $N^{*}$ pertenecientes a $N$ y que $I$ $\in A(\mathrm{~N} *)$;

(5) $p$ sabe en $t$ que la expansión de $I$ en $t$ necesariamente debilita la red $N$ y que el refinamiento de $N$ en $t$ disminuye el rango de aplicaciones intencionales;

(6) $p$ cree que existen refinamientos de $N$ aplicables no sólo a $I$, sino incluso a conjuntos, que incluyen I.” (Stegmüller 1978, pp. 50-51)

Nuevamente, las condiciones (1) y (2) se refieren a la origen histórico de la teoría; (3) expresa la idea de continuidad histórica; mientras que la condición (4) describe el conocimiento empírico y las aserciones empíricas de aquellos que disponen de una teoría; (5) refleja la idea de que alguien que dispone de una teoría sabe que expandir el conjunto de aplicaciones intencionales, "debilita" la red $N$, esto implica el abandono ciertas especializaciones (ciertas leyes especiales y restricciones especiales) utilizadas en la construcción de $N$; mientras que quien dispone de una teoría también sabe de la posibilidad de "refinar" una red, esto es, ensamblar especializaciones adicionales dentro de la red; (6) expone la creencia de las personas en el progreso de la ciencia normal, donde sumar especializaciones a la red, produce mejoras adicionales a ésta, y a su vez, expansiones mayores del conjunto $I$. 
Stegmüller agrega a continuación de la (Def. III), en consonancia con las apreciaciones de Stegmüller $(1973,1976)$, que una versión extendida del concepto de "disponer de teoría" mejora la comprensión del concepto kuhniano de ciencia normal. De modo que, varias personas pertenecen a la misma tradición de ciencia normal, solo si disponen de la misma teoría en el sentido caracterizado arriba.

3.2.3. Finalmente, podemos agregar que, dados los cambios en la conceptualización metateórica mencionados en (3.2.2), cuando Stegmüller (1979) volvía a comentar su interpretación de Kuhn, en los años transcurridos se habían introducido precisiones en el ámbito del desarrollo de la CE, en particular con los aportes de Sneed, J. (1976), Balzer, Sneed (1977, 1978), Moulines, Sneed (1979). Por otro lado, las interpretaciones de Stegmüller sobre Kuhn (y también sobre Lakatos) habían sido objeto de varias críticas $^{11}$, y ambos sucesos provocaron que Stegmüller revisara algunas de sus afirmaciones sobre su integración de estos autores a la concepción estructural de la dinámica de las teorías.

Stegmüller (1976) ya comenzaba a acercar la idea de cambio científico con la idea de evolución histórica progresiva en el estructuralismo ${ }^{12}$, posición que será más desarrollada en Stegmüller (1979). Aquí, se concibe la dinámica científica como evolución teórica, siendo esta noción la que el autor encuentra más próxima a la idea de programa de investigación de Lakatos, y a su vez más propicia para dar cuenta de la idea de evolución en un período de ciencia normal.

Stegmüller (1979) reformulaba su interpretación de Kuhn, afirmando que: "Los aspectos dinámicos pueden ser también analizados sin hacer uso del concepto de disponer de una teoría. Esto puede ser hecho por medio del concepto de red, enriquecido pragmáticamente.” (Stegmüller 1979, p.123).

Así, en Stegmüller (1979) la noción general de "evolución teórica" de una red teórica pasaba a ser una alternativa de análisis a la idea de actos de "disponer de una teoría", a los fines de explicar el desarrollo de una teoría científica en un período de ciencia normal. En éste un grupo de científicos operan dentro de un paradigma, y la dinámica del mismo implica el

${ }^{11}$ De particular importancia resultó Tuomela, R. (1978).

12 "En ocasiones Lakatos habla de la teoría de Einstein o de la de Newton. En tales contextos la palabra 'teoría' se usa aparentemente en el sentido de 'programa de investigación'. Con esto en mente y detalles menores aparte, llegamos al resultado de que su concepto de programa de investigación progresivo coincide con el concepto de evolución teórica progresiva en el sentido usado aquí.” Stegmüller (1976, p. 84). 
desarrollo de una red, donde el curso normal, progresivo de ésta, se basa en sucesivas confirmaciones, que fijan los elementos paradigmáticos de $I$ y las ampliaciones sucesivas de dicho conjunto. La nueva consideración no abandonaba, según el autor, la orientación pragmática del tratamiento de la dinámica de la ciencia, sino que introducía ahora aspectos pragmáticos de la confirmación, como acciones intervinientes en los procesos de cambio teórico intraparadigmáticos, o cambios en los períodos de ciencia normal kuhnianos.

\section{La CE: concepción de teoría y componentes pragmáticos. Una integra- ción con la propuesta kuhniana. Segundos desarrollos}

4.1. En continuación con los desarrollos que había emprendido Stegmüller, Moulines (1982, 1991a, 1991b, 1994, 2004) ahondará la vía de análisis de componentes pragmáticos de la ciencia.

En Moulines (1982) se reconocía que en la noción de paradigma kuhniano hay elementos que escapan al análisis tradicional de teoría, siendo uno de los principales, la inclusión de la práctica científica en el análisis de la ciencia. Sostiene: "Para identificar una teoría física no basta con captar su estructura formal especificada en el núcleo, sino que además hay que reconocer sus aplicaciones paradigmáticas. Esto responde a la idea kuhniana de que parte de las aplicaciones de una teoría son indisolubles de la noción misma de teoría”. (Moulines 1982, p. 85)

El autor afirma: "Creo que todos estos aspectos semánticos y pragmáticos adicionales de las teorías empíricas se pueden resumir alrededor de la noción clave de aplicación de una teoría. El análisis del concepto de aplicación resulta, pues, central para la filosofía de la ciencia.” Moulines (1982, p.56). Seguidamente, agrega:

“Algunos filósofos (entre ellos quizá Suppes) probablemente piensan que, aunque el concepto de aplicación va típicamente asociado a las teorías empíricas, no pertenece realmente a las últimas y que, por tanto, podemos separar la tarea de reconstruir las teorías particulares de la tarea de aclarar la noción de aplicación. Pero no creo que esto sea metodológicamente aceptable. Las teorías científicas están siempre construidas en vistas a algunas aplicaciones «externas» concretas, de tal modo que las aplicaciones están incluidas también conceptualmente en la teoría misma." Moulines (1982, p.57).

Moulines propone, al modo en que muchos autores hablan de la carga teórica de las observaciones empíricas, hablar de la «carga aplicativa» de todos los constructos teóricos. Además, las teorías científicas no tienen un 
conjunto limitado y estático de aplicaciones, por el contario, sostiene el autor:

"Esta multiplicidad de aplicaciones...constituyen un conjunto abierto en el sentido de que no se puede determinar extensionalmente de una vez por todas. Depende de consideraciones pragmáticas, las cuales, lo mismo, que cualquier otro aspecto pragmático de la ciencia, cambian en el transcurso del tiempo e incluso según el usuario". [...] "Así, pues, una teoría resulta ser una entidad esencialmente determinada no sólo por su estructura formal y por su referencia, sino también por su uso." Moulines (1982, p.58)

Como consecuencia de la valoración del rol y la determinación sociohistórica y pragmática del conjunto de las aplicaciones intencionales, desde los aportes de Moulines (1982) se incorporarán a las definiciones tanto de elemento teórico, como de red teórica las nociones de "comunidades científicas" $\left(C C_{i}\right)$ e "intervalos históricos" $\left(h_{i}\right)$; así como la relación $\leq$, que indica: "es anterior o simultáneo a...". Dentro de estas nociones, la de comunidad científica es la que requiere de más precisión. Al respecto, Moulines explicita que la noción de CC implica que: (i) es un grupo de personas que dura más de una generación (una comunidad científica es una entidad genidéntica); (ii) Los miembros del grupo se comunican entre sí en un "lenguaje científico" específico; (iii) Todos ellos participan de técnicas de medición específicas y de procedimientos observacionales y de cálculo para contrastar sus hipótesis. (Moulines 1982, p.113).

A partir de lo anterior, tendremos que un elemento teórico $T=\langle K, I$, $C C, h>$, donde, como antes, $K$ es el núcleo de $T$; $I$ es el conjunto de aplicaciones intencionales, $C C$ es una comunidad científica, y $h$ un intervalo histórico. A esto se agrega las precisiones de que $I \subseteq \operatorname{Pot}(M p p)$; y que CC se propone aplicar $K$ a $I$ durante $h$.

Asimismo, la definición de una red teórica $R$ implicará que: (1) $R$ sea un conjunto de elementos teóricos; (2) $R$ esté parcialmente ordenado por la relación de especialización teórica; (3) que para cada par de elementos teóricos $T_{i}, T_{j} \in R, C C_{i}=C C_{i}, \mathrm{y}(4)$ para cada par de elementos teóricos $T_{i}$, $T_{j} \in R, h_{i}=h_{j}^{j}$

La definición de red teórica $R$ (enriquecida pragmáticamente) mencionada arriba caracteriza la red de modo estático, e implica que en un mismo período histórico (sincrónicamente considerado), los defensores de los elementos teóricos, que pertenecen a una misma red $R$, son miembros de una misma comunidad científica. Se incorpora, de modo análogo al conjunto $I$ de un elemento teórico, el conjunto $I(R)$ de la totalidad de dominios de aplicaciones correspondientes a los elementos teóricos de $R$, donde $I(R)$ es equivalente a $\cup Q(R), y Q(R)=\{I / \exists T(T \in R \wedge T=<K, I, C C, h>\}$. 
4.2. En Moulines (1991a, 1991b, 1994) la propuesta sigue sus conceptualizaciones de Moulines (1982) y se incorpora la noción de "generación científica" $(G)$. Esta noción es equivalente a un corte sincrónico de la comunidad científica $(C C)$. La identidad de una $G$ consiste en grupos de usuarios coetáneos de determinados elementos teóricos $T_{i}$ que pretenden aplicar los modelos propios de $T_{i}$ a ciertos sistemas físicos o fenoménicos. Estos usuarios -sostiene Moulines- tienen una determinada actitud proposicional con respecto a dichos elementos modelo-teóricos. Si consideramos $E=<M p p$, $M>$ un elemento teórico, e $I$ a un subconjunto de $M p p$, entonces la actitud proposicional en cuestión se expresa como: " $G$ intenta aplicar $E$ a I". (Moulines, 1991a, 284)

Según nuestro autor, ésta es la actitud proposicional básica según el análisis pragmático-diacrónico de la ciencia propugnado por el estructuralismo, e implica que $G$ (y no sus miembros individualmente tomados) constituye el sujeto epistémico de la actitud proposicional es cuestión. Esto es así, ya que los miembros de $G$ tienen entre sí determinadas relaciones, principalmente la de comunicación en un lenguaje técnico propio, la de cooperación, según ciertos procedimientos metódicos (por ejemplo los de observación, clasificación, medición, experimentación, etc.) y las de competencia y negociación de acuerdo a ciertos patrones de conducta (lucha por el apoyo financiero o de otro tipo por parte de las instancias externas, peer review, estrategias de citas mutuas, etc.).

Además, cuando afirmamos que " $G$ intenta aplicar $T$ a $I$ " en dicha aplicación debemos distinguir la existencia de un subconjunto de $I,\left(I_{b}\right)$ que constituye el conjunto de aplicaciones confirmadas por $G$. Formalmente, la proposición " $I_{b} \subset r(M)$ " es considerada bien confirmada (donde " $I_{b} \subset$ $r(M)$ " significa que el conjunto $I_{b}$ está incluido dentro del "reducto de M", o sea del conjunto de todos los modelos potenciales parciales que pueden extenderse a modelos actuales). A su vez, habrá un subconjunto $I_{b}$ el conjunto $I_{p}$, de "aplicaciones intencionales paradigmáticas", que $G$ estima metodológicamente central, y que constituye justamente una referencia paradigmática para el resto de los casos de aplicación. Los elementos de ese conjunto corresponden —según Moulines- a lo que Kuhn (1962) ha descrito como "ejemplos compartidos". El conjunto $I_{p}$ resulta altamente relevante en el enfoque, y en su determinación no puede recurrirse a ningún criterio formal; sino a aspectos histórico-pragmáticos, que conducen a indagar acerca de cómo nació la teoría, cómo evolucionó, qué aplicaciones sirvieron de ejemplos característicos y permanentes, para hallar nuevas aplicaciones a partir de relaciones de semejanza con aquellos. $G$ funciona en relación a 
$I_{p}$ con una actitud proposicional particular, como relación parcial de intención de aplicación, formulada del siguiente modo: " $G$ reconoce a $I_{p}$ como paradigma para I”. Así, se introduce la siguiente definición:

Def. IV:

"Los miembros de $G$ comunican, cooperan y compiten entre sí con el propósito de mostrar que para un cierto elemento modelo teórico $E=<M_{p}, M>$ y para un cierto subconjunto $I \subset M_{p p}$ se cumple $I \subset r(M)$; y para un subconjunto $I_{b} \subset I$ esto ya está confirmado". Moulines (1991, p.287).

La definición anterior deja en claro la interrelación estrecha entre $G$ y la determinación del conjunto $I$. Las acciones de los miembros de una generación consolidan el conjunto de aplicaciones intencionales, a lo largo del desarrollo de una red, y lo amplían. Partiendo del subconjunto $I_{p}$ de ejemplos paradigmáticos de $I$, éste se va consolidando en el transcurso del tiempo, sobre la base de las confirmaciones que fijan $I_{b}$ y permiten el crecimiento sucesivo de $I$.

4.3. Hasta aquí, me he limitado a considerar los aspectos pragmáticos de la CE, que han entrado en consonancia con categorías propias de la filosofía de la ciencia kuhniana. En este último punto en lo atinente a la obra de Moulines, haré una referencia rápida a los logros más recientes, saliéndome parcialmente del objetivo inicial, a los efectos de evaluar posteriormente el estado de general de la cuestión.

En varias obras aproximadamente contemporáneas al texto de Moulines (1991a) comentado (Moulines 1991b, 1994) se reiteran, con leves variaciones, las consideraciones pragmáticas sobre la ciencia vertidas en aquel texto. Sin embargo, en Moulines (2002) ${ }^{13}$ se amplían y desarrollan nuevos tópicos en relación a la temática citada. En este texto se presenta un conjunto de aspectos en que la pragmática "se agazapa" desde la lectura metateórica de la CE. El primero de esos aspectos es el tradicional tópico de las aplicaciones intencionales. Se reiteran ideas ya trabajadas, respecto de la labor de los miembros de una comunidad científica CC, quienes realizan las aplicaciones del núcleo teórico. Estos miembros son conceptualizados en términos de "sujetos epistémicos", y por ende, sujetos que sostienen determinadas actitudes proposicionales. La definición respectiva es la siguiente: "CC intenta o se propone subsumir ciertos elementos de $M p p$ (de $K$ ) bajo la porción $T$-teórica de $K$ (modelos actuales, ligaduras y vínculos)", donde ese

${ }^{13}$ El texto de Moulines (2002) corresponde al trabajo presentado en el I Encuentro Internacional "Perspectivas actuales de la metateoría estructuralista", celebrado en Zacatecas, México, 1998; publicado en Diez, J.A. y Lorenzano, P. (eds.) (2002). 
conjunto de elementos de $M p p$ a los que se aplica la actitud proposicional, constituye el conjunto de aplicaciones intencionales $(I): I=\{i: i \in M p p \wedge C C$ intenta subsumir $i$ bajo $K$ \} (Moulines, 2002, p. 102).

De los restantes componentes pragmáticos a los que Moulines (2002) hace referencia, se encuentran los intervinientes en la determinación de los términos $T$-teóricos y $T$-no-teóricos. Este tópico es uno de los más trabajados en el marco de la CE, desde la formulación del criterio de teoricidad de Sneed (1971). No solo Moulines, sino, entre otros Balzer (1996) han enfatizado que dichos criterios supone el uso de expresiones tales como "determinación de un término" y "presuposición de $T$ " en una determinación de un término. Tales expresiones implican considerar la existencia de una teoría dada $T$ y de un grupo de científicos que operan con $T$. Para la obtención, por ejemplo, de algunos valores de ciertas funciones de $T$ para ciertos argumentos, se requiere de acciones como experimentos y mediciones que se llevan a cabo en orden a determinar los valores de aquellas. Estas actividades representan las determinaciones para algún término. Teniendo en cuenta ejemplos concretos de éstas, reconocemos que, en el curso de estos procesos, los científicos en general usan conocimiento teórico, fórmulas, ecuaciones. Éstos realizan ciertos cálculos e inferencias sobre la base de fórmulas dadas de modo de obtener valores de la función que se desea conocer. Usualmente, este conocimiento teórico se da en un contexto concreto sin mayor justificación: es (hipotéticamente) asumido o presupuesto. Todos los tipos de asunciones teóricas usadas en el curso de una determinación son presuposiciones. Así, en este marco metateórico, para la determinación de un término (una ecuación, una fórmula, etc.) hay dos casos posibles: o se da que proviene (es parte de) alguna teorías $T$ ' diferente de $T$ (y en ese caso es $T$-no-teórico) o es parte de, o idéntica a $T$, de modo que coincide con los axiomas de $T$ (y en ese caso es $T$-teórico). ${ }^{14}$

Hasta aquí, no se manifiesta novedad en el planteo, pero lo que sigue en el texto, sí propone perspectivas no contempladas previamente. Estas consideraciones sobre la pragmática de la ciencia parten de la reflexión sobre la aserción empírica central de una teoría $T,(I \in C n(K))$. El autor nos recuerda que desde Moulines (1976) se ha enfatizad la necesidad de acudir a la idea de "aproximaciones admisibles" (A), ya que ninguna teoría logra ser aplicada con toda exactitud a sus aplicaciones propuestas,

${ }^{14}$ He considerado aquí parcialmente la elucidación de (Balzer, 1996) sobre los aspectos pragmáticos del criterio de teoricidad de Sneed, que forman parte del trabajo: Gonzalo, A. "Criterios de $T$-teoricidad y el problema del significado de los términos científicos en la Concepción Estructural de la ciencia”. Workshop sobre la T-teoricidad en la metateoría estructuralista. Universidad Nacional de Quilmes. Buenos Aires, 2009. 
sino que lo hace en una versión aproximativa de la aserción empírica: $I \in \sim$ $\mathrm{Cn}(K)$; de este modo, sería conveniente simbolizar $T=\langle K, A, I>$. $A$ se ha considerado tradicionalmente ${ }^{15}$ como un subconjunto de la uniformidad empírica $U$, definida sobre $M p: U \subseteq \operatorname{Pot}\left(M p{ }^{\prime} M p\right)$. Si bien esta definición no parece acarrear aspectos pragmáticos, el autor asume que al momento de introducir el conjunto $A$, se deben determinar los grados de aproximación admisibles. Aquí los miembros de $C C$ deben ejercer particiones de $I$, de acuerdo a ciertos criterios propios de las prácticas científicas de CC (según la determinación de resultados más precisos, que hagan uso de instrumentos más sofisticados, según las valoraciones de comprobación asumidas, y de impacto (económico, tecnológico, etc.) de las aplicaciones propuestas). De modo que la determinación de $A$, implica la selección de un subconjunto admisible de $I$, con los señalados aspectos pragmáticos involucrados.

Los otros dos aspectos pragmáticos señalados en Moulines (2002), son aquellos atinentes a la determinación de los componentes mismos de $K$ : los concernientes a distinción entre $M p$ y $M$; y el concerniente a los vínculos interteóricos. En relación al primero de éstos, el problema se centra en la posibilidad de determinar si ciertos postulados, llamados unirrelacionales son estimados por la CC como leyes empíricas (y pertenecen por ende a $M$ ) o caracterizaciones de las relaciones (y en ese caso, pertenecen a $\mathrm{Mp}$ ). Los criterios comunitarios llevarían consigo en la práctica la decisión, en los procedimientos de validación, y consecuente tareas de aceptación o rechazo de las teorías. El segundo se refiere a la posibilidad de determinar entre vínculos esenciales entre dos teorías $T$ y $T^{\prime}$, y vínculos no-esenciales; siendo los primeros los que constituyen los $M$ de la teoría. Por diversas razones expuestas en el texto, las distinciones semánticas entre vínculos determinantes e implicativos, - propias de la bibliografía tradicional de la CE - no resultan suficientes para la determinación de la esencialidad aludida; y la misma depende, en muchos casos, de las tradiciones científicas y del uso mismo de la teoría.

A partir de las últimas consideraciones, vemos como se amplía considerablemente la agenda de problemas metateóricos relativos a la elucidación de los aspectos pragmáticos en la CE. Como hemos comentado, algunos de éstos eran ya muy conocidos y desarrollados en el marco de la CE, pero Moulines (2002) logra llamar la atención acerca de aquellos que en principio no se explicitan, pero que quedan presupuestos aún en los componentes teóricos más formales de una teoría empírica. ${ }^{16}$

${ }^{15}$ Balzer, W., Moulines, C.U., Sneed. J. (1987)

${ }^{16}$ La lista propuesta por Moulines (2004) podría constituir un programa de trabajo en la línea de una visión más pragmática de la $\mathrm{CE}$, pero no conozco trabajos que hayan avanzado en esa dirección en el seno de los desarrollos posteriores de la CE. 


\section{Evaluación de las propuestas de integración entre Kuhn y la CE sobre los componentes pragmáticos de la ciencia.}

Uno de los aspectos claramente conciliables entre los enfoques de la CE y Kuhn, al que nos hemos referido, es la concepción de teoría. Al respecto, ambas posiciones se enfrentan a la visión enunciativa de la ciencia. La idea de aplicaciones intencionales, como propias de la definición misma de teoría en la $\mathrm{CE}$, se muestra muy próxima a la atención a las prácticas científicas dadas en el seno de las comunidades científicas en la conceptualización kuhniana. En particular, el rol de la idea de aplicaciones paradigmáticas, resulta análoga a la idea de ejemplares, y resalta un aspecto central de la pragmática de la actividad científica.

A pesar de lo anterior, es también claro que la CE se aproxima siempre a la noción de aplicaciones intencionales desde una perspectiva en la que una teoría se piensa básicamente en relación a la posibilidad de hacer aserciones acerca de X, donde X debe "modelarse" según T, (aunque al mismo tiempo debe poder ser de algún modo modelable en un lenguaje diferente a $T)$. Disponer de una teoría $T$ está, por ende, asociado a la idea de realizar aserciones empíricas de $T$. Visto así, la caracterización pragmática de esta disponibilidad de $T$ se halla aún bastante circunscripta a una imagen de la ciencia como actividad asertórica, y una función básicamente cognitiva.

Lo dicho también se constata en la reiteración de la expresión "comunidad epistémica", para referirse a la comunidad científica; como también en el uso sistemático de la expresión "actitudes proposicionales" (sobre la cual se insiste en su carácter propio de la filosofía del lenguaje), para referirse a las actividades propias de la CC.

Asimismo, cuando Stegmüller estima que la elección de los ejemplos paradigmáticos, como aplicaciones intencionales de los miembros de un grupo de científicos, se da sobre evidencia empírica, sin duda, limita bastante del planteo kuhniano. Justamente, esta afirmación conlleva un aspecto restrictivo al estimar la evidencia empírica como incorporando racionalidad a la creencia del científico, y por ende a su elección. Es sobre la base de disponer de evidencia observacional que — según Stegmüller- la persona "cree que" y realiza ciertas aserciones empíricas sobre un conjunto de sistemas físicos.

En la misma línea que Stegmüller, Moulines habla de "confirmación" y de "estándares de confirmación” para que una generación científica ejercite ciertas actitudes proposicionales, en relación al conjunto de aplicaciones intencionales de una teoría. La misma tendencia puede apreciarse cuando Moulines (1991a) habla de "comunicación", "cooperación” y "competencia y negociación” como elementos de la práctica científica. Éstas se dan 
sobre la base de que " $I_{b} \subset I$ está ya confirmada". Del mismo modo, cuando se habla de comunicación, se menciona "en un determinado lenguaje técnico", y de cooperación: "según procedimientos metódicos (observación, clasificación, medición, experimentación, etc.). Resumidamente, la práctica científica se interpreta, casi siempre, en términos de accionar según pautas metodológicas precisas, comunicarse según un lenguaje técnico claro, realizar elecciones y aserciones sobre la base de confirmaciones empíricas. En este sentido, se considera que se "cierra la fisura irracional kuhniana".

La expresión "cerrar la fisura irracional de Kuhn", presente en muchas ocasiones en las obras de Sneed, Stegmüller y Moulines hace también referencia a la idea kuhniana de cambio científico. Desde la CE se intentaba "normalizar" la teoría kuhniana de cambio revolucionario, inicialmente a través de la idea de reducción, noción trabajada ya por Sneed, que fuese luego redefinida en Stegmüller $(1973,1976)$ y otros autores. Como hemos señalado, en Stegmüller (1979) se dan algunos cambios centrales: (i) la disminución del rol central de la noción de "disponer de una teoría", en contraposición al protagonismo relevante que comienza a tomar la noción de evolución teórica como herramienta conceptual para la reconstrucción diacrónica de la ciencia; (ii) la interpretación de falsacionismo sofisticado y su relación con la idea de reducción postulada por Stegmüller (1973), pasar a ser una relación formal, marco de relaciones interteoréticas precisas entre teorías (redes teóricas). En contraposición a la conceptualización propuesta en Stegmüller $(1973,1976)$, la CE caracterizará posteriormente el cambio teórico — centralmente, aunque no excluyentemente- en términos del crecimiento de una red teórica y la de evolución teórica en un período de ciencia normal. ${ }^{17}$

Por otro lado, es claro que, el estructuralismo no ha desarrollado una metateoría que explique en un sentido histórico-pragmático el cambio

${ }^{17}$ Las limitaciones de restringir la idea de "cambio teórico" a la de "evolución teórica" han sido expuestas dentro mismo de la CE. Así, por ejemplo, en los últimos años, algunos trabajos de reconstrucción diacrónica de las teorías han mostrado que la idea de programa de investigación de Lakatos podría interpretarse en términos de Holon, siguiendo la conceptualización llevada a cabo en Balzer, Moulines, Sneed (1987, pp. 387-390). Asimismo, en Zoubek, G. y Lauth, B. (1992 a y b) se marca lo restrictivo que resulta hablar de redes teóricas como unidades del desarrollo de la ciencia dentro de la concepción de evolución teórica, y se ha propuesto interpretar los elementos teóricos como unidades intraprogramáticas. Además, se ha pensado que la idea de reducción resulta adecuada para interpretar las relaciones que se establecen entre los elementos teóricos que integran el programa de investigación. La noción de reducción es concebida allí como una relación interteorética entre elementos teóricos, y hasta el momento esta nueva visión del cambio intra-programático no ha incorporado una conceptualización socio-histórica. 
revolucionario en ciencia. La idea de reducción de teorías, y un gran conjunto de ideas que analizan las relaciones entre teorías, han sido propuestas como relaciones interteoréticas, y su conceptualización responde a un enfoque formal de las relaciones entre teorías. Como hemos señalado, el mismo Kuhn, que aceptó con agrado gran parte de la interpretación de su filosofía realizada por el estructuralismo, no concibió que éste ofreciese una reconstrucción de su idea de cambio revolucionario ${ }^{18}$; y en verdad, en la CE la noción ha sido generalmente interpretada en términos de aquella fisura irracional a saldar.

En lo que respecta a la elección teórica que ejercen los actores de las comunidades científicas, en el marco del estructuralismo, ésta se presenta, en casi todos los casos, regida por criterios metodológicos y procedimientos basados en la evidencia empírica disponible. Pero, recordemos que Kuhn ha insistido sistemáticamente sobre la existencia de criterios de elección teórica, solo que interpretados como normas de acción comunitaria, y en muchos casos también transcomunitarias. ${ }^{19}$ En el tratamiento del tema entraban en discusión problemas metodológicos tradicionales relativos a la elección teórica, pero también la mostración de casos ejemplares de la historia de la ciencia en los que los criterios lógicos y metodológicos no lograban explicar la persistencia de creencias o la elección teórica en casos determinados. En su defecto, la teoría del cambio teórico se explicaba sobre la idea de aceptación o rechazo involucrando criterios del orden pragmático. Éstos incorporaban aspectos del orden de las prácticas comunicacionales, que incluyen tanto aspectos relativos a la semántica de los términos y expresiones de las teorías así como formas retóricas y técnicas persuasivas, que llevarían a la "conversión teórica" —idea tan irritante para muchos de la comunidad filosófica-. ${ }^{20}$

Finalmente, es dable mencionar que el guante lanzado por Kuhn a los filósofos de la ciencia, de tomar en consideración casos concretos de historia de la ciencia para las reconstrucciones y reflexiones metateóricas, fue claramente recogido por la CE, desde donde el estudio de casos ha resultado

${ }^{18}$ Kuhn se refiere en muchos de sus textos a estos aciertos y limitaciones de la CE para captar plenamente su posición. En particular en el citado Kuhn (1976); en el prefacio de Kuhn (1977); en Kuhn (1981) y Kuhn (1983).

${ }^{19}$ Particularmente en Kuhn (1976).

${ }^{20}$ En relación a lo anterior, el texto de Moulines (2004) ofrece una vía alternativa a lo comentado en referencia a las propuestas dentro de la CE, y abre la discusión a la intervención de aspectos claramente pragmáticos vinculados a la elección teórica en campo de la ciencia empírica. 
parte central del minucioso trabajo epistemológico de reconstrucciones de la dinámica de la ciencia. ${ }^{21}$

\section{Consideraciones Finales}

Al comienzo del trabajo nos hemos referido a la "historia heredada" de la filosofía de la ciencia, que ha sostenido la idea de ruptura entre un período prekuhniano y uno postkuhniano, a partir de una lectura del girosociohistórico. Esta lectura presentaba este giro como demarcante de dos enfoques antagónicos: enfoques formales o lógico-semánticos de la ciencia, y enfoques socio-históricos.

Como se mencionó en la introducción, un propósito que perseguíamos era oponernos a dicha visión histórica. La estrategia seguida partía de revisar la noción de teoría presupuesta, que atribuye a los enfoques formales una concepción enunciativa de teoría: la teoría concebida como conjunto de enunciados, lógicamente estructurados, que refieren a un campo particular del dominio físico de objetos y cuyo fin es realizar explicaciones y pre-

${ }^{21}$ Actualmente existe un vastísimo número de ejemplos de reconstrucción en las ciencias físico-natulares, muchos de los cuales han sido recogidos en Balzer, Moulines y Sneed (1987); Balzer y Moulines (1996); Balzer, Moulines y Sneed (ed.) 2000. Asimismo, en el campo de las ciencias sociales encontramos numerosas reconstrucciones: Balzer; W. (1982) "The Proper Reconstruction of Exchange Economics" Erkenntnis 17, pp. 23-46; Haslinger, F. (1983) "A Logical Reconstruction of Pure Exchange Economics: An Alternative View" Erkenntnis, 20, pp. 115-129; Diederich, W. "The Development of Marx's Economic Theory". Erkenntnis Vol. 30, 1-2, pp. 147-164; García de la Sienra, A.G.(1982)"The Basic Core of the Marxian Economic Theory", en Stegmüller. Balzer y Spohn (eds.) pp. 118144. En psicología existen también una rica cantidad de ejemplos, como Westmeyer, H. (ed.), Psychological Theories From a Structuralist Point of View, New York: Springer, 1989; Westmeyer, H. (ed.), The Structuralist Program in Psychology: Foundations and Applications, Göttingen: Hogrefe \& Huber, 1992. En lingüística: Gonzalo, A. (2001) Cambios modeloteóricos en la lingüistica chomskiana. Una reconstrucción desde la concepción estructural de la ciencia. Tesis Doctoral, Universidad de Buenos Aires. Peris Viñé, L. M. (1990) "First Steps on the Reconstruction of Chomskyan Grammar”, en Diez, A., Echeverría, J. et al. (eds) Structures in Mathematical Theories. Universidad del País Vasco, pp. 83-87; Peris Viñé, L. M. (1991) "Relación de especialización, gramática universal y gramáticas particulares”, en Martín Vide, C. (ed.) Lenguajes Naturales y Lenguajes Formales VI, Barcelona, Publicaciones Universitarias, pp. 839-859; Quesada, D. (1993). "Grammar a Theory: An Analysis of the Standar Model of Syntax Within the Structural Programm”, en Diez, A; Echeverría, J. et al. (eds) Structures in Mathematical Theories, Universidad del País Vasco, pp. 1751982; Balzer, W. y Gonzalo, A. "Eine strukturalistische Rekonstruktion einer linguistischen Theorie. Volume in Honor of Prof. Petros Gemtos. Athens: Ant. N. Sakkoulas Publishers. (en prensa: 2009). 
dicciones mediante dicho aparato teórico-conceptual. En contraposición, hemos mostrado en gran parte del análisis que la noción de teoría de la CE conlleva aspectos pragmáticos de la ciencia, constitutivos de la noción de teoría, y hemos resaltado al respecto el rol del concepto de aplicaciones intencionales.

Esta visión de teoría de la CE se acerca a la posición kuhniana, en relación a noción de teoría como paradigma; y en particular, es dable de interpretar nociones como la de ejemplares kuhnianos en el marco de dicha concepción, como aplicaciones paradigmáticas. El énfasis en el rol de los componentes pragmáticos, tanto como de los componentes lógico-semánticos y herramientas conjuntistas en la reconstrucción de teorías, se contrapone a la imagen habitual dicotómica entre enfoques sociológicos y formales de la ciencia.

Asimismo, las preocupaciones desde Sneed (1971) por captar la dinámica de las teorías en las reconstrucciones estructuralistas, rompe con la antinomia: enfoques estáticos estructurales; versus enfoques histórico-sociales. Los desarrollos posteriores, tanto en Stegmüller (1973, 1976, 1978, 1979), en Moulines (1982, 1991a, 1991b, 1994), como los desarrollos en Balzer, Moulines, Sneed (1987); Balzer, Moulines (1996); Balzer, Sneed, Moulines (2000) representan esfuerzos múltiples y constantes en el objetivo de atrapar la dinámica del cambio teórico. Estos intentos han tenido, casi permanentemente, a Kuhn como un interlocutor protagónico.

A pesar de lo anterior, y en consonancia con la evaluación realizada en el apartado (5) el diálogo con Kuhn desde la CE ha insistido sistemáticamente en la idea de "cerrar la fisura irracional kuhniana". Un aspecto de la misma parecía constituirla la vaguedad e imprecisión propias del planteo kuhniano. Una organización definicional, y sobre todo una reconstrucción formal parecerían remediales de estas falencias. La confianza en la claridad y precisión del aparato formal de la CE era puesto en marcha en la caracterización y definición del conjunto de elementos básicos de las reconstrucciones de las teorías científicas.

La recuperación del componente de análisis pragmático, a través de la consideración de acciones de los miembros de la comunidad científica, permitía el análisis del actor de la comunidad científica kuhniana (racionalizado por el estructuralismo) como un sujeto que "cree" en la posibilidad de realizar aplicaciones exitosas de $K$ a sistemas físicos $X$, a partir de un conjunto ejemplar de aplicaciones exitosas. Además, este miembro puede creer en la posibilidad de extender éstas, ampliando el conjunto de aplicaciones exitosas a partir de ciertas especializaciones hechas sobre las leyes fundamentales del núcleo, y configurar un conjunto más restringido y exitoso 
de aplicaciones intencionalmente dadas en un campo empírico concreto. Pero, es claro que en la lectura de la CE, las acciones de estos miembros, ha dejado fuera de la agenda epistemológica a muchos componentes centrales del análisis de las comunidades científicas de T. Kuhn. Sin embargo, podría objetarse que, no se trata de subsumir el enfoque kuhniano dentro de la CE, sino de hacerlo conciliable en los límites propuestos por este enfoque.

Concluyendo, agregaría que los trabajos analizados aquí — salvo el comentado en (4.3) - se remiten centralmente a los desarrollos de la CE hasta la década del noventa. El camino posterior de la CE ha seguido centralmente una vía de clarificación metateórica, de refinamiento en las herramientas de formalización; así como de ampliación del campo de las reconstrucciones en muy diversas áreas de la ciencia empírica. Esta vía, que si bien ayudó a ganar en precisión, y aumentó la fecundidad de la metateoría en análisis de casos; fue lentamente dejando sin profundizar las nociones pragmáticas programáticamente introducidas en las primeras décadas de su desarrollo. Como se ha señalado ya, la excepción en este camino ha sido la obra de Moulines, C. U. (1991a, 1991b, 1994, 2004), donde la agenda problemática trazada queda abierta a desarrollos futuros y a continuar los ideales conciliatorios de Stegmüller entre los enfoques formales y los históricopragmáticos en la filosofía de la ciencia.

\section{Referencias Bibiográficas}

Balzer, W. (1996) “Theorical Terms: Recent Developments”, en Balzer, W. y Moulines, C.U, Structuralist Theory of Science, Berlín, W. de Gruyter, 1996.

Balzer, W., Moulines, C. U., Sneed, J. (1987) An Architectonic for Science. Dordrecht, Reidel.

Balzer, W., Moulines, C.U. (eds.) (1996) Structuralist Theory of Science. Berlín, W. de Gruyter.

Balzer, W., Sneed, J. y Moulines, C. U, (eds.) (2000) Structuralist Knowledge Representation. Paradigmatic Examples. Amsterdan, Rodopi.

Balzer, M. y Sneed, J.D. (1977) "Generalized Net Structures in Empirical Sciences I". Studia Logica 36, 195-211.

Balzer, M. y Sneed, J.D. (1978) "Generalized Net Structures in Empirical Sciences II”. Studia Logica 37, 167-194.

Barnes, B. (1976) Interest and the Growth of Knowledge, Londres, Routledge and Kegan, 
Bloor, D. (1976) Knowledge and Social Imagenery. Londres, Routledge and Kegan.

Brown, H. (1977) Perpection, Theory and Commitment. The New Philosophy of Sciencie. University Illinois Press.

Diederich, W.; Ibarra. A. et.al. (1989) "Bibliography of Structuralism 19711988". Erkenntnis 30, 387-407.

Diederich, W.; Ibarra. A. et al. (1994). "Bibliography of Structuralism II 1989-1994 and Additions". Erkenntnis 41, 403-418.

Diez, J.A. y Lorenzano, P. (Eds.) Desarrollos actuales de la metateoría estructiralista: problemas y discusiones. Buenos Aires, Universidad Nacional de Quilmes, 2002.

Falguera, J. L. (1999) "Ontosemantic Divergence and Comparability of Theories". Logica Trianguli. 3, 33-53.

_ (2004) "Las revoluciones científicas y el problema de la inconmensurabilidad", en González, W. J. (ed.) Análisis de Thomas Kubn: Las revoluciones científicas. Madrid, Trotta, 177-223.

_ (2009) "De lo que tratan en común teorías inconmensurables". Discusiones Filosóficas. Año 9 No 12, 13-35.

Fleck, L. (1935) La génesis y el desarrollo de un hecho científico. Madrid, Alianza, 1980.

Jaramillo, J. M. (1996) “De la ontosemántica de los términos y enunciados a la ontosemántica de las teorías", en Filosofía y Ciencia. Ed. Universidad del Valle, Colciencias, Sgo de Calis, Colombia, 159-188.

Kuhn, T. (1962) La estructura de las revoluciones científicas. México, FCE, 1975.

(1969) "Posdata", en La estructura de las revoluciones científicas. México, FCE, 1975.

_ (1971) "Segundas reflexiones sobre paradigmas", en Suppe, F. (comp.) La estructura de las teorías científicas. Madrid, Editora Nacional, 1979.

_ (1976) "Theory Change as Structure-Change: Comments on the Sneed Formalism", Erkenntnis 10, 179-199.

_ (1977) La tensión esencial. México, FCE, 1982.

_ (1983) "Conmensurabilidad, comparabilidad y comunicabilidad", en Kuhn, T. ¿Qué son las revoluciones cientificas? y otros ensayos. Barcelona, Paidós, 1989, 95-135.

_ (2000) El camino desde la estructura. Ensayos filosóficos 1970-1993. Conant, J. y Haugeland, J. (Comps.). Barcelona, Paidós, 2002.

Hacking, I. (1981) "Introduction", en Hacking, I. (ed.) Scientific Revolutions. Oxford University Press, Oxford, 1981. 
Hesse, Mary (1980) Revolutions and reconstructions in the philosophy of science, Brighton, The Harvester Press.

Lakatos, I. (1970) "Falsification and the Methodology of Scientific Research Programmes", en Lakatos, I, y Musgrave, A. (eds.) Criticism and the Growth of Knowledge, Londres. Cambridge University Press, 1970, 99196.

(1971) "History of Science and his Rational reconstruction", en Buck R. and Cohen, R. (eds.) Boston Studies in the Phisosophy of Science, Vol. VIII, Humanities Press, New York, 1972, 91-136.

Lorenzano, C. y Lorenzano, P. (1996) "En memoria de Thomas S. Kuhn”. Redes. Nro 7, pp.217-236.

Lorenzano, P. (2008) "Inconmensurabilidad teórica y comparabilidad empírica: el caso de la genética clásica”. Análisis Filosófico, XXVIII Nro 2, 239-279.

Moulines, C. U. (1982) Exploraciones metacientíficas. Madrid, Alianza.

_ (1991a) Pluralidad y recursion. Estudios Epistemológicos. Madrid, Alianza.

_ (1991b) "Pragmatics in the Structralist View of Science", en Shurz, G. and Dorn, G.J.W. (Comps.) Advances in Scientific Philosophy. Amsterdam, Rodopi, 1991, 313-326.

_ (1994) "Wer bestimmt was es gibt?”. Zeitschrift für Philosophische Forschung 48, 2, 175-191.

_ (2002) “¿Dónde se agazapa la pragmática en la representación estructural de las teorías?”, en Diez, J.A. y Lorenzano, P. (Eds.) (2002), 99-115.

- (2010) "Metatheoretical Structuralism: a General program for Analyzing Science". Axiomathes 20, 255-268.

Moulines, C. U., Sneed, J. (1979) "Suppes' Philosophy of Physics", en Patrick Suppes, Bogdan, R. (comp.) Dordrecht. Reidel, 59-92.

Pérez Ransanz, A. R. (1999) Kubn y el cambio científico. México, FCE.

Putnam, H, (1962) "What Theories are not", en Nagel, E., Suppes, P y Tarski, A. (dir.) Logic, Methodology and Philosophy of Science. Stanford, 1962, 240-251.

Rossi, P. (1986) Las arañas y las hormigas. Una apología de la Historia de la Ciencia. Barcelona, Crítica, 1990.

Shapere, D. "Meaning and Scientific Change", en Colofny R, (ed.) Mind and Cosmos: Essays in Contemporany Science and Philosophy. University of Pittsburg Press, 41-85.

Sneed, J. (1971) The Logical Structure of Mathematical Physics. Dordrecht, Reidel. 
_ (1976) "Philosophical Problems in the Empirical Science of Science: A Formal Approach". Erkenntnis 10, 115-146

Stegmüller, W. (1973) Probleme und Resultate der Wissenschaftstheorie und Analytischen Philosophie.Vol. II: Theorie and Erfahrung, Sub-volumen II: Theorienstrukturen und Theoriendynamik. Heidelberg, Springer. Versión utilizada: Estructura y Dinámica de las Teorías. (Trad. C. U. Moulines) Barcelona, Ariel, 1983.

_ (1976) “Accidental ('Non Substancial') Theory Change and Theory Dislodgement: to What Extent Logic can contribute to a Better Understanding of Certain Phenomena in Dynamics of Theories". Erkenntnis 10, 147-178. Versión utilizada: "Cambio teórico accidental (no sustancial) y desplazamiento de teorías”, en A.A.V.V. Estructura y desarrollo de las teorías cientificas. México, UNAM, 1986.

_ (1978) "A Combined Approach to the Dynamics of Theories. How to Improve Historical Interpretations of Theory Change by Applying Set Theoretical Structures". Theory and Decision 9, 39-75.

_ (1979) "The Structural View: Survey, Recent Developments, and Answers to some Criticisms", en Niniluoto, I. y Tuomela R. (eds.): The Logical and Epistemology of scientific change, (Acta Philosophica Fennica) North-Holland Publ. Co.

Suppe, F. (1974) "What's Wrong with the Received View on the Structure of Scientific theories?", en Philosophy of Science 2, 1-19.

Tuomela, R.(1978) "On the Structuralist Approach to the Dynamics of Theories". Synthese 39, 211-231.

Zoubek, G. y Lauth, B. (1992 ${ }_{\mathrm{a}}$ ) "Zur Rekonstruktion des Bohrschen Forschungsprogramms I". Erkenntnis 37, 223-247.

Zoubek, G. y Lauth, B. (1992 $)$ "Zur Rekonstruktion des Bohrschen Forschungsprogramms II”. Erkenntnis 37, 249-273. 